\title{
Deconvolution closure for mesoscopic continuum models of particle
} systems

\author{
Alexander Panchenko ${ }^{1}$, Lyudmyla Barannyk ${ }^{2}$, and Kevin Cooper ${ }^{1}$ \\ ${ }^{1}$ Washington State University \\ ${ }^{2}$ University of Idaho
}

February 21, 2022

\begin{abstract}
We present a framework for derivation of closed-form continuum equations governing mesoscale dynamics of large particle systems. Balance equations for spatial averages such as density, linear momentum, and energy were previously derived by a number of authors. These equations are exact, but are not in closed form because the stress and the heat flux (e.g. stress in the momentum balance equation) cannot be evaluated without the knowledge of particle positions and velocities. Recently, we proposed a method for approximating exact fluxes by true constitutive equations, that is, using non-local operators acting only on the average density and velocity. In the paper, constitute operators are obtained by using filtered regularization methods from the theory of ill-posed problems. We also formulate conditions on fluctuation statistics which permit approximating these operators by local equations. The performance of the method is tested numerically using Fermi-Pasta-Ulam particle chains with two different potentials: the classical Lennard-Jones, and the purely repulsive potential used in granular materials modeling. The initial conditions incorporate velocity fluctuations on scales that are smaller than the size of the averaging window. Simulation results show good agreement between the exact stress and its closed form approximation.
\end{abstract}

\section{Hosted file}

deconvolution_closure_MMAS.pdf available at https://authorea.com/users/461692/articles/ 557320-deconvolution-closure-for-mesoscopic-continuum-models-of-particle-systems
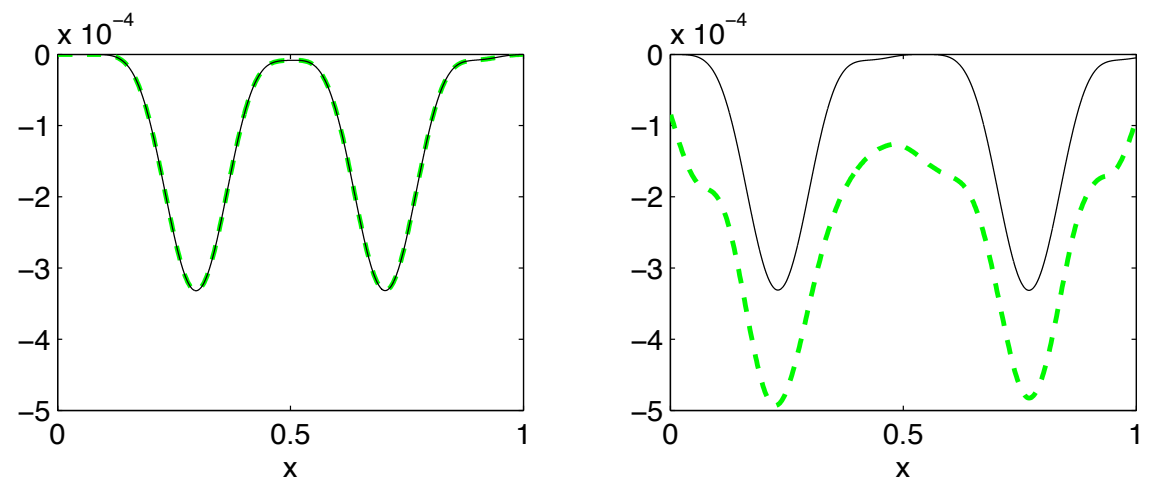

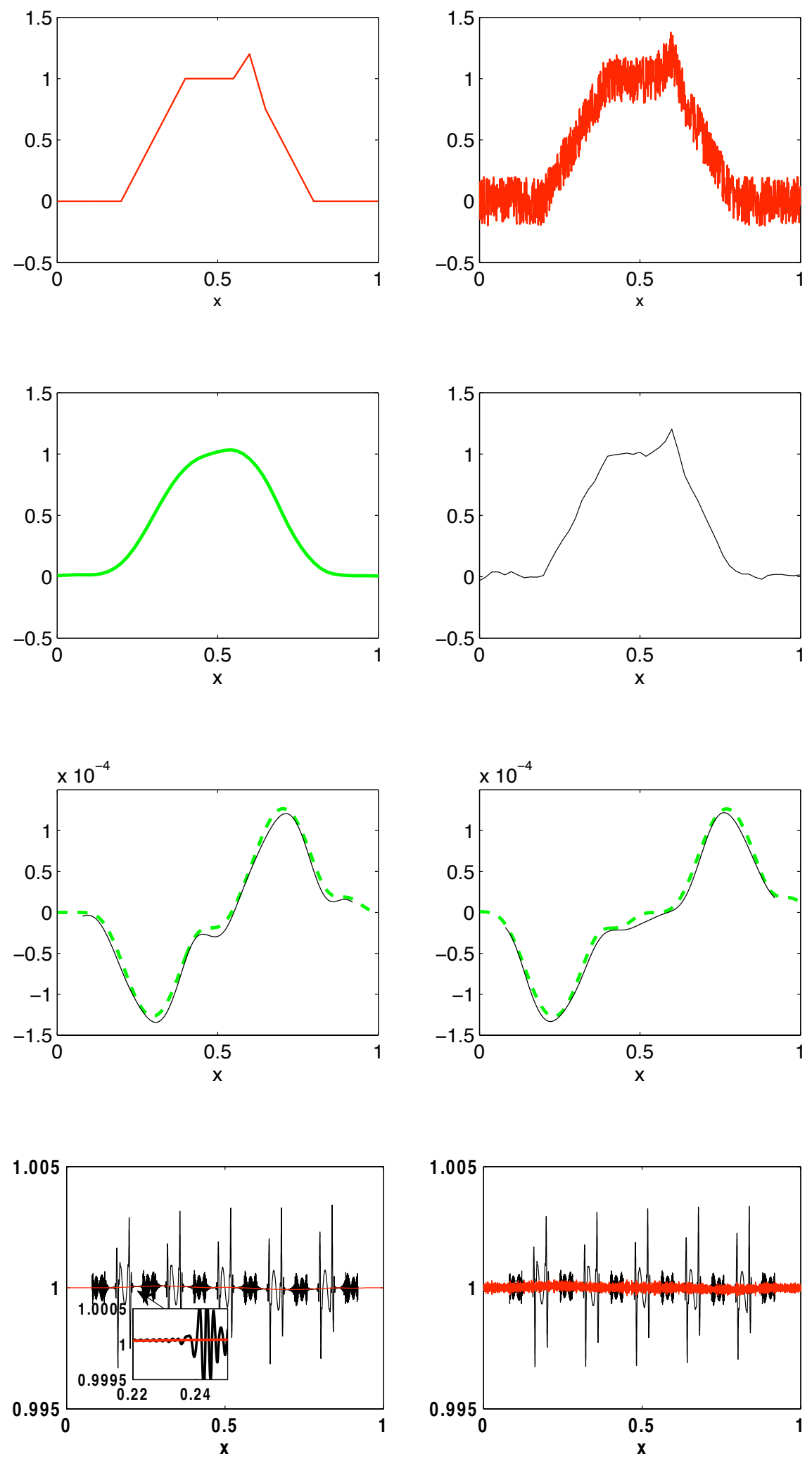

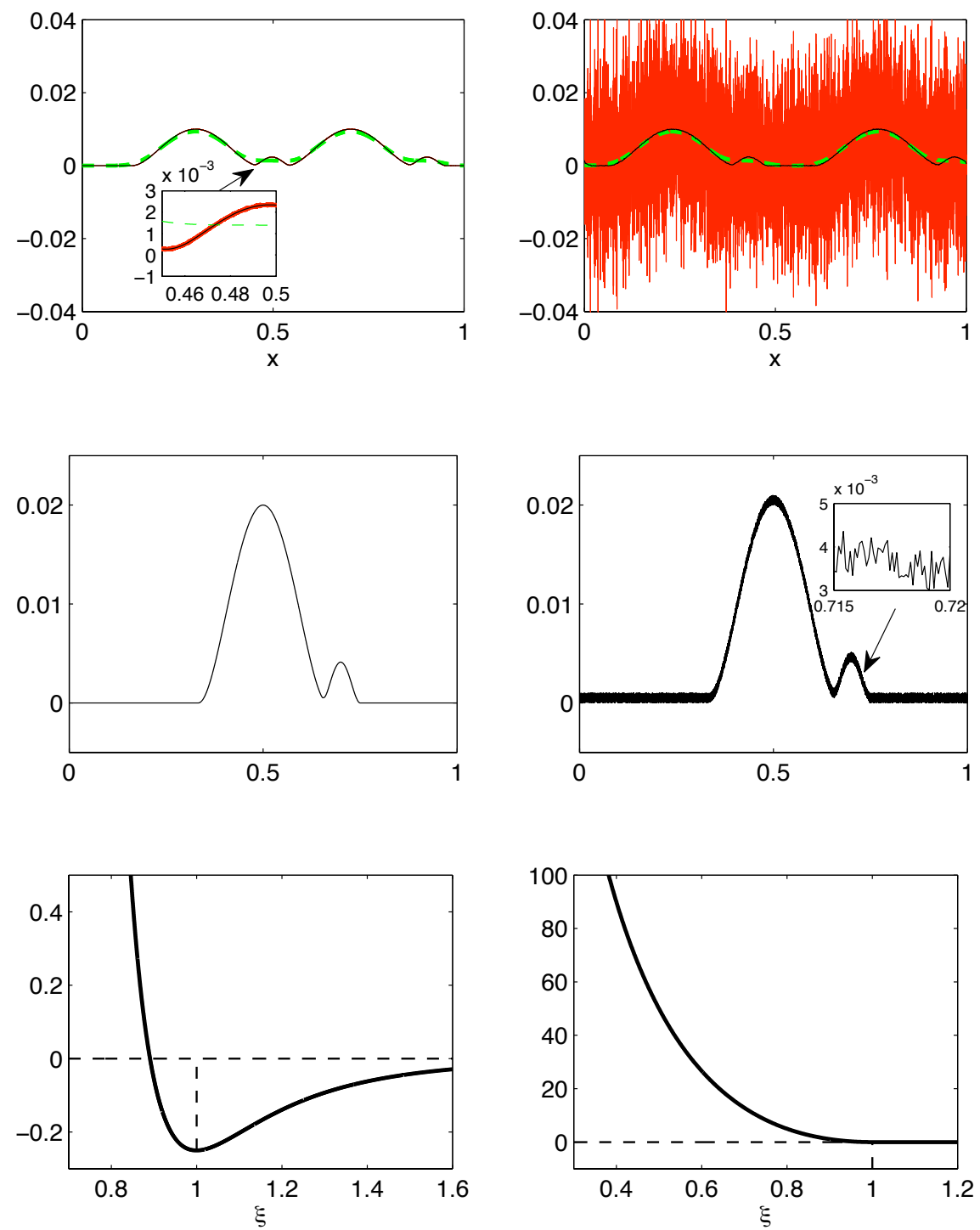

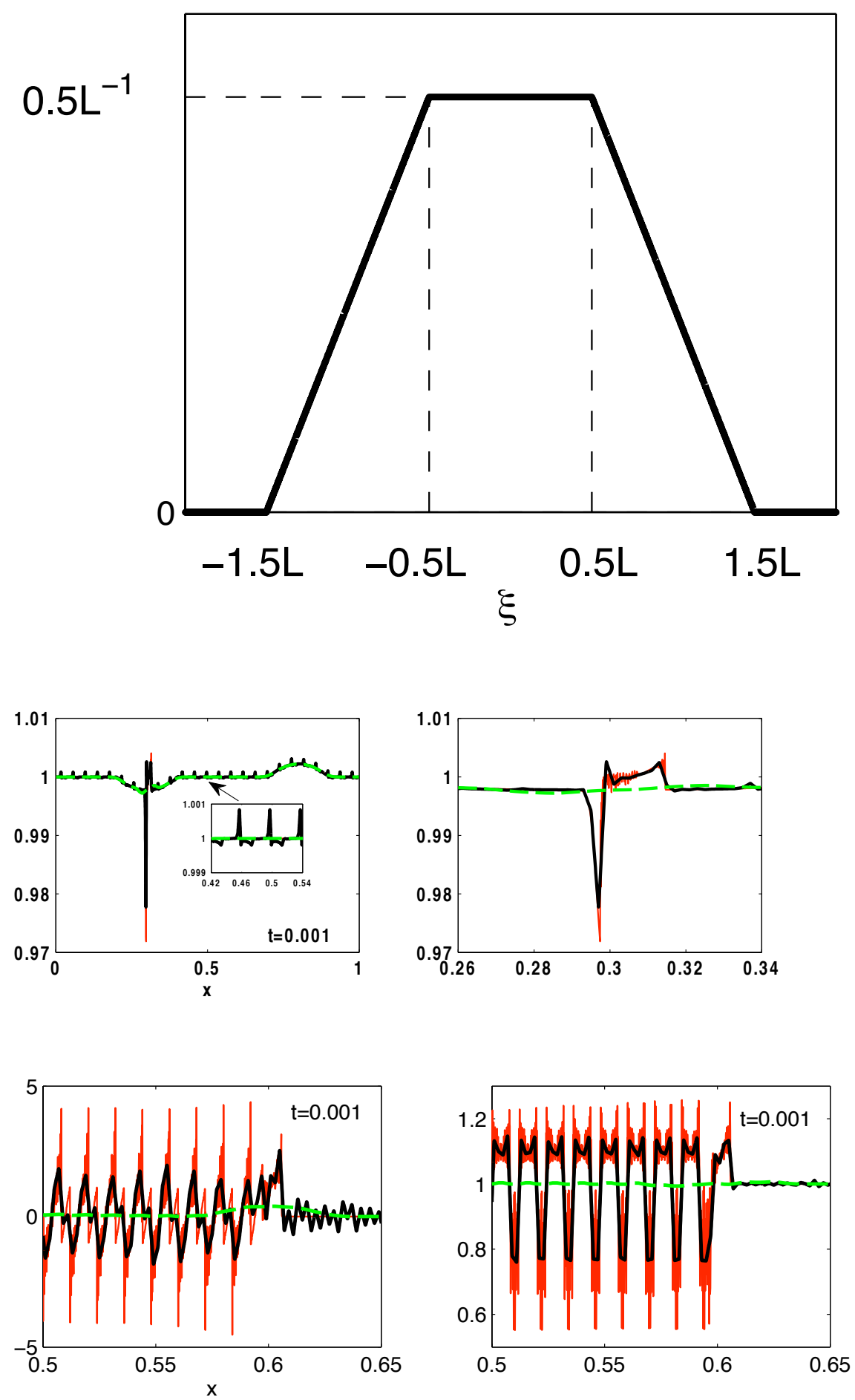

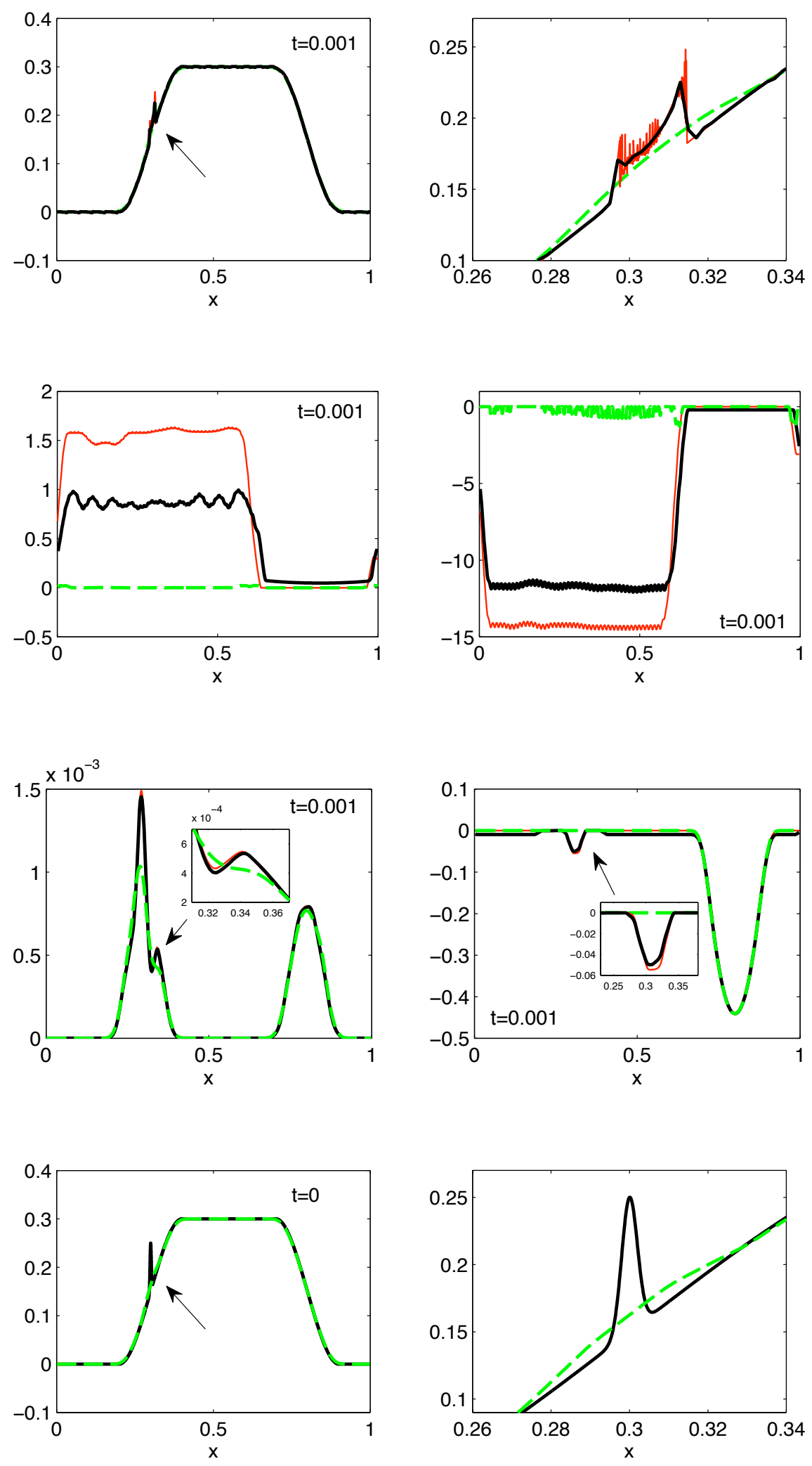

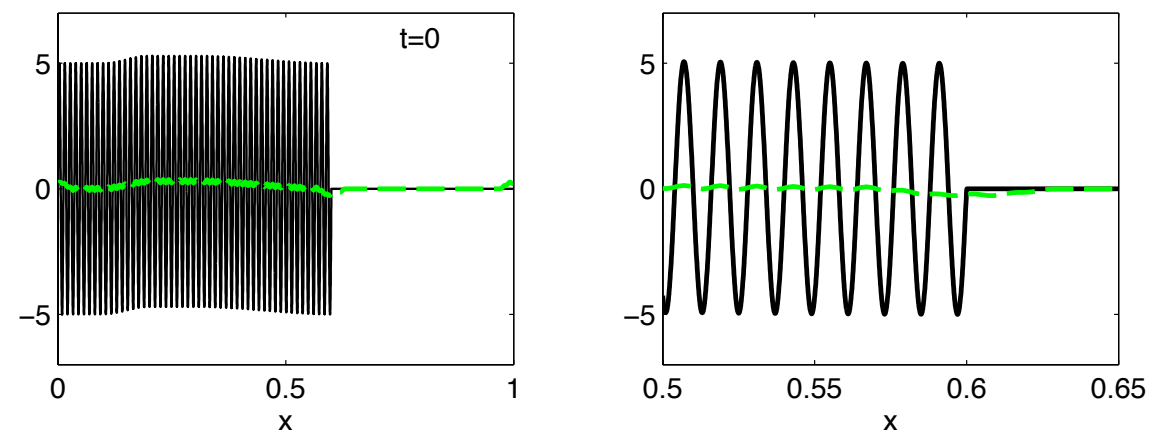